\title{
Liquiritin Relieves Oxygen-Glucose Reperfusion-Induced Neuronal Injury via Inhibition of the p38MAPK/NF-KB Signaling Pathway
}

\author{
Liangying Liao ${ }^{1}$ (D) Zhanwei Zhang $^{2}$ (D) \\ Received: 19 October 2021 / Accepted: 12 January 2022 / Published online: 1 March 2022 \\ (C) The Author(s) 2022
}

\begin{abstract}
In traditional Chinese medicine, liquiritin, an active component of Glycyrrhiza uralensis Fisch., Fabaceae, has several pharmacological effects such as anticancer, antioxidant, and neuroprotective properties. The present study aimed to explore the protective functions and molecular mechanisms underlying the effects of liquiritin on nerve injury induced by cerebral ischemia/ reperfusion. SH-SY5Y cells were incubated with varying concentrations of liquiritin for different periods of time, and 3-(45)dimethylthiahiazo(-z-y1)-35-di-phenytetrazoliumromide and lactate dehydrogenase assays were employed to determine the levels of cell viability and damage. Subsequently, cells were exposed to oxygen and glucose deprivation/reoxygenation to establish an ischemia/reperfusion injury model. The results revealed that liquiritin protected SH-SY5Y cells from oxygen and glucose deprivation/reoxygenation-induced damage by improving viability and reducing apoptosis, and oxidative stress. Liquiritin inhibited activation of the p38 mitogen-activated protein kinase (MAPK)/nuclear factor kappa B (NF-KB) signaling pathway. In addition, treatment with a p38MAPK-specific agonist reversed the protective effects of liquiritin.
\end{abstract}

Keywords Cerebral ischemia/reperfusion injury · Chinese liquorice $\cdot$ Flavanone $\cdot$ Glycyrrhiza radix $\cdot$ Neuronal damage Oxidative damage

\section{Introduction}

Ischemic stroke is a leading cause of death and disability worldwide, and the associated clinical prognosis remains poor $(\mathrm{He}$ et al. 2014). Early reperfusion following cerebral ischemia is key for brain survival and functional recovery (Liao et al. 2020). Prompt restoration of blood circulation following ischemic stroke reduces the impact of cerebral ischemia injury; however, the exposure of ischemic tissue to blood oxygen may result in cerebral ischemia/reperfusion (I/R) injury (Liu et al. 2020; Xu et al. 2020). Cerebral I/R injury is associated with high rates of disability, morbidity, and mortality and remains a complex issue

Zhanwei Zhang

20202022@stu.hnucm.edu.cn

1 Scientific and Research Department, First Affiliated Hospital, Hunan University of Chinese Medicine, Changsha, Hunan 410007, People's Republic of China

2 Department of Neurosurgery, First Affiliated Hospital, Hunan University of Chinese Medicine, Changsha, Hunan 410007, People's Republic of China within stroke therapy (Meng et al. 2019). Previous research has focused on the mortality of vulnerable neurons in ischemic brain damage (Liu et al. 2017). Also, several neuroprotective methods have remained a key focus within the therapy of cerebral $\mathrm{I} / \mathrm{R}$ damage (Abdel-Rahman et al. 2020). Results from a previous study reported that oxidative stress and neuronal apoptosis were associated with cerebral I/R injury (Zhang and Zhang 2020). Inflammation is also a key pathogenic characteristic of cerebral $\mathrm{I} / \mathrm{R}$ injury, resulting in secondary brain injury and deterioration of the central nervous system (Geng et al. 2020). Thus, further investigations are required to identify novel medical treatments with antioxidant, antiapoptotic, or anti-inflammatory properties to serve as neuroprotective agents for the treatment of cerebral I/ $\mathrm{R}$ injury.

In traditional Chinese medicine (TCM), natural components have been tested for their efficacy in the treatment of several human metabolic diseases. Glycyrrhiza uralensis Fisch., Fabaceae, also known as Chinese liquorice, is used as a sweetener and in TCM. Liquorice root, or Glycyrrhiza radix, is used in Chinese medicine to treat coughs, reduce phlegm, and relieve depression (Zhou et al. 2020). Liquiritin (1) is a major flavonoid isolated from this medicinal plant ( $\mathrm{Li}$ 
et al. 2020a), which corresponds to the $4^{\prime}$ - $O$-glucoside of the flavanone liquiritigenin, (2S)-4',7-dihydroxyflavan-4-one.

Results from a previous study reported that liquiritin possesses a number of pharmacological properties, including antiinflammatory, antioxidant, and neuroprotective properties (Zhang et al. 2016). Moreover, previous research has revealed that liquiritin may exert antioxidative and antiapoptotic effects in brain injury caused by cerebral I/R injury (Jia et al. 2016). Results from a previous study demonstrated that liquiritin improved tissue injury via inhibition of MAPK and toll-like receptor 4/myeloid differentiation factor 88 signaling pathways, which reduced the associated inflammatory response (Li et al. 2018). However, the molecular mechanisms underlying the protective effects of liquiritin in cerebral I/R injury are yet to be fully investigated.

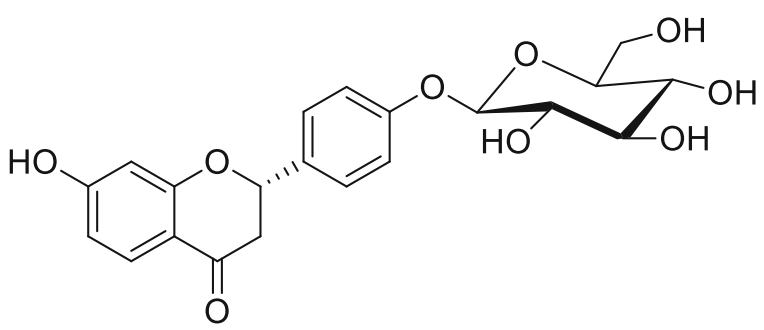

1

$\mathrm{NF}-\kappa \mathrm{B}$, a key transcriptional activator in inflammation, activates pro-inflammatory cytokines following nuclear translocation. Notably, I/R injury induces NF- $\mathrm{B}$ B activation (Xie et al. 2019). NF-KB is closely associated with MAPK, and NF- $K B$ activation may be mediated by MAPK in cerebral I/ $\mathrm{R}$ injury (Li et al. 2020b). Thus, it was hypothesized that liquiritin may exert protective effects in cerebral $I / R$ injury via the $\mathrm{p} 38 \mathrm{MAPK} / \mathrm{NF}-\mathrm{kB}$ signaling pathway.

In the present study, an in vitro oxygen and glucose deprivation/reoxygenation (OGD/R) model was established to determine the protective effects of liquiritin (1) on cell viability, apoptosis, and antioxidant enzyme activities. In addition, the role of the $\mathrm{p} 38 \mathrm{MAPK} / \mathrm{NF}-\mathrm{kB}$ pathway was also investigated in cerebral I/R injury.

\section{Materials and Methods}

\section{Cell Cultures}

Human neuronal SH-SY5Y cells were purchased from the American Type Culture Collection. Cells were cultured in Dulbecco's modified Eagle medium (DMEM) (HyClone; Cytiva) containing 10\% FBS (Gibco; Thermo Fisher Scientific, Inc., Waltham, MA, USA) and $1 \%$ penicillin/streptomycin (Thermo Fisher Scientific, Inc.), and maintained at $37{ }^{\circ} \mathrm{C}$ with $5 \% \mathrm{CO}_{2}$. The cell culture medium was changed regularly. The toxic effects of liquiritin (1) (Chengdu Conbon Biotech Co. Ltd., Chengdu, China) on the SH-SY5Y cell were tested with different concentrations of liquiritin $(0,1.25,2.5,5,10,20,40,80$, and $160 \mu \mathrm{M})$ at $37^{\circ} \mathrm{C}$ for $24 \mathrm{~h}$.

\section{OGD/R Establishment}

Briefly, SH-SY5Y cells were plated into 96-well plates and cultured for $24 \mathrm{~h}$. Subsequently, cells were cultured in glucose-free DMEM with $94 \% \mathrm{~N}_{2}, 5 \% \mathrm{CO}_{2}$, and $1 \% \mathrm{O}_{2}$, and incubated for $3 \mathrm{~h}$ at $37^{\circ} \mathrm{C}$. In addition, cells were reoxygenated for $24 \mathrm{~h}$ in DMEM in a normoxic incubation chamber at $37{ }^{\circ} \mathrm{C}$. Control cells were cultured at $37{ }^{\circ} \mathrm{C}$ with $5 \% \mathrm{CO}_{2}$. To study the effect of liquiritin on OGD/R-induced SH-SY5Y cell damage, $\mathrm{SH}$ SY5Y cells were incubated in an anaerobic gas mixture $(1 \%$ $\mathrm{O}_{2}, 5 \% \mathrm{CO}_{2}$, and $94 \% \mathrm{~N}_{2}$ ) at $37{ }^{\circ} \mathrm{C}$ in the presence or absence of liquiritin (20, 40, $80 \mu \mathrm{M})$ and p38/MAPK agonist (P79350; $50 \mu \mathrm{M}$ ). After $3 \mathrm{~h}$, the cells were restored from the anaerobic chamber to a normoxic environment, and then oxygenated for 24 h. Subsequently, the following experiments were performed.

\section{Cell Viability}

Briefly, $25 \mu$ l of MTT reagent was added to cells and cultured for $3 \mathrm{~h}$ at $37^{\circ} \mathrm{C}$. Subsequently, the supernatant was removed, and $200 \mu \mathrm{l}$ dimethyl sulfoxide was added to solubilize the formazan crystals. The optical density was measured at $570 \mathrm{~nm}$ using a VersaMax ELISA microplate reader.

\section{Lactate Dehydrogenase Leakage}

Lactate dehydrogenase (LDH) leakage was detected using the cytotoxicity assay. A total of $1 \times 10^{4}$ cells were seeded into 96-well plates and incubated with different conditions. After treatment, the $\mathrm{LDH}$ release reagent was added to cells and incubated for $1 \mathrm{~h}$ at $37^{\circ} \mathrm{C}$. The absorbance was measured using a microplate reader at $490 \mathrm{~nm}$.

\section{ELISA}

The enzyme-linked immunosorbent assay (ELISA) was performed to examine the expression levels of TNF- $\alpha$, IL- $1 \beta$, and IL-6 in cell supernatants using a specific ELISA kit (Beyotime Institute of Biotechnology Co., Ltd., Nantong, Jiangsu, China), according to the manufacturer's protocol.

\section{Flow Cytometry}

To determine the levels of cell apoptosis, an Annexin V-FITC/ PI kit (BD Biosciences) was used. Briefly, cells were washed twice with cold PBS. Subsequently, a total of $1 \times 10^{5}$ cells were collected in $200 \mu \mathrm{l}$ binding buffer, $5 \mu \mathrm{PI}$, and $10 \mu \mathrm{l}$ Annexin V-FITC, and incubated in the dark for $15 \mathrm{~min}$. After 
Fig. 1 Effects of liquiritin (1) on the viability of SH-SY5Y cells.

SH-SY5Y cells were treated with varying concentrations of liquiritin $(0,1.25,2.5,5,10,20$, $40,80$, and $160 \mu \mathrm{M})$ for $24 \mathrm{~h}$. Cell viability $(\mathbf{A})$ and LDH activity $(\mathbf{B})$ were investigated using MTT and LDH assays, respectively. Data are presented as the mean $\pm \mathrm{SD}$. Abbreviation: $\mathrm{LDH}$, lactate dehydrogenase

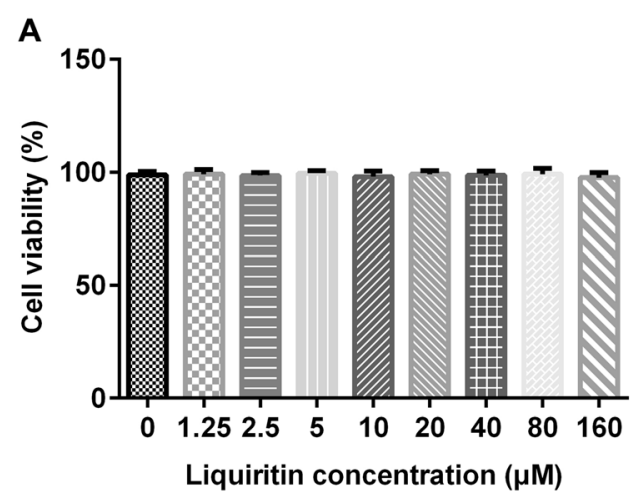

staining, FACSCalibur was used to detect the fluorescence intensity.

\section{Antioxidant Enzymatic Activities}

Briefly, SH-SY5Y cells were treated with different conditions. Following treatment, indicators of antioxidant enzyme activity, including superoxide dismutase (SOD), catalase (CAT),

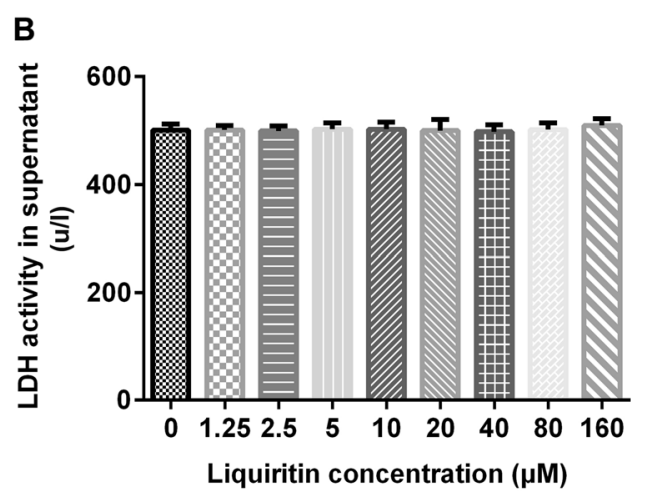

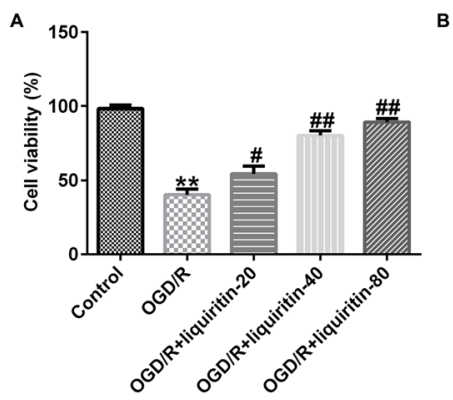

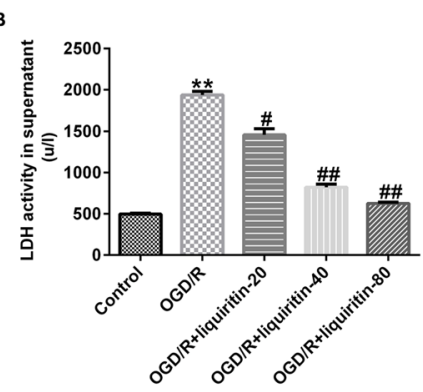

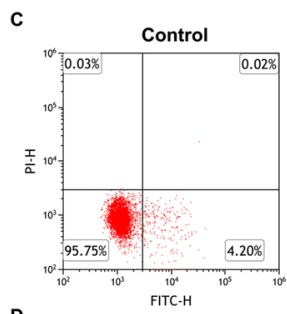
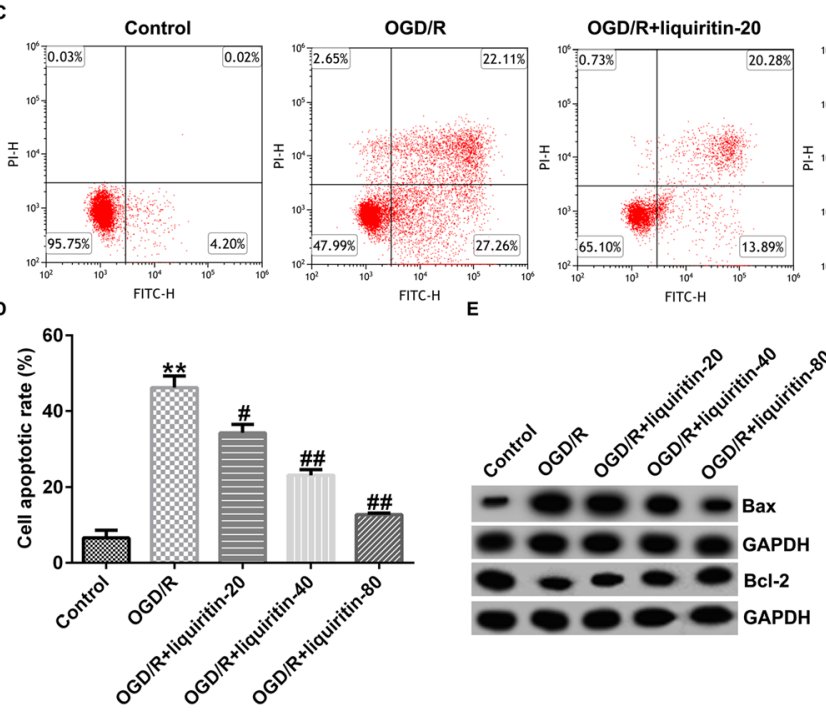

Fig. 2 Liquiritin (1) increases survival and reduces apoptosis of OGD/Rinduced SH-SY5Y cells. SH-SY5Y cells were treated with varying concentrations of liquiritin $(0,20,40$, and $80 \mu \mathrm{M})$, followed by $\mathrm{OGD} / \mathrm{R}$ treatment. Cell viability $(\mathbf{A})$ and $\mathrm{LDH}$ activity $(\mathbf{B})$ were investigated using MTT and LDH assays, respectively. (C and D) Cell apoptosis was determined using flow cytometry assays. (E) Western blot analyses were used glutathione peroxidase (GSH-Px), and MDA, were detected by using commercially available kits (Sigma-Aldrich; Merck $\mathrm{KGaA}$ ), according to the manufacturer's protocol.

\section{Western Blot Analysis}

Total protein was extracted from SH-SY5Y cells using RIPA lysis buffer (Beyotime Institute of Biotechnology) on ice for 30 
Fig. 3 Liquiritin (1) inhibits OGD/R-induced oxidative stress in SH-SY5Y cells. (A-D) Indicators of antioxidant enzyme activity, such as SOD, CAT, and GSH-Px, and MDA expression levels were detected using commercially available kits. Data are presented as the mean $\pm \mathrm{SD}$. ${ }^{* *} p<$ 0.01 vs. control; ${ }^{\#} p<0.05,{ }^{\# \#} p<$ 0.01 vs. OGD/R. Abbreviations: $\mathrm{OGD} / \mathrm{R}$, oxygen and glucose deprivation/reoxygenation; SOD, superoxide dismutase; CAT, catalase; GSH-Px, glutathione peroxidase; MDA, malondialdehyde
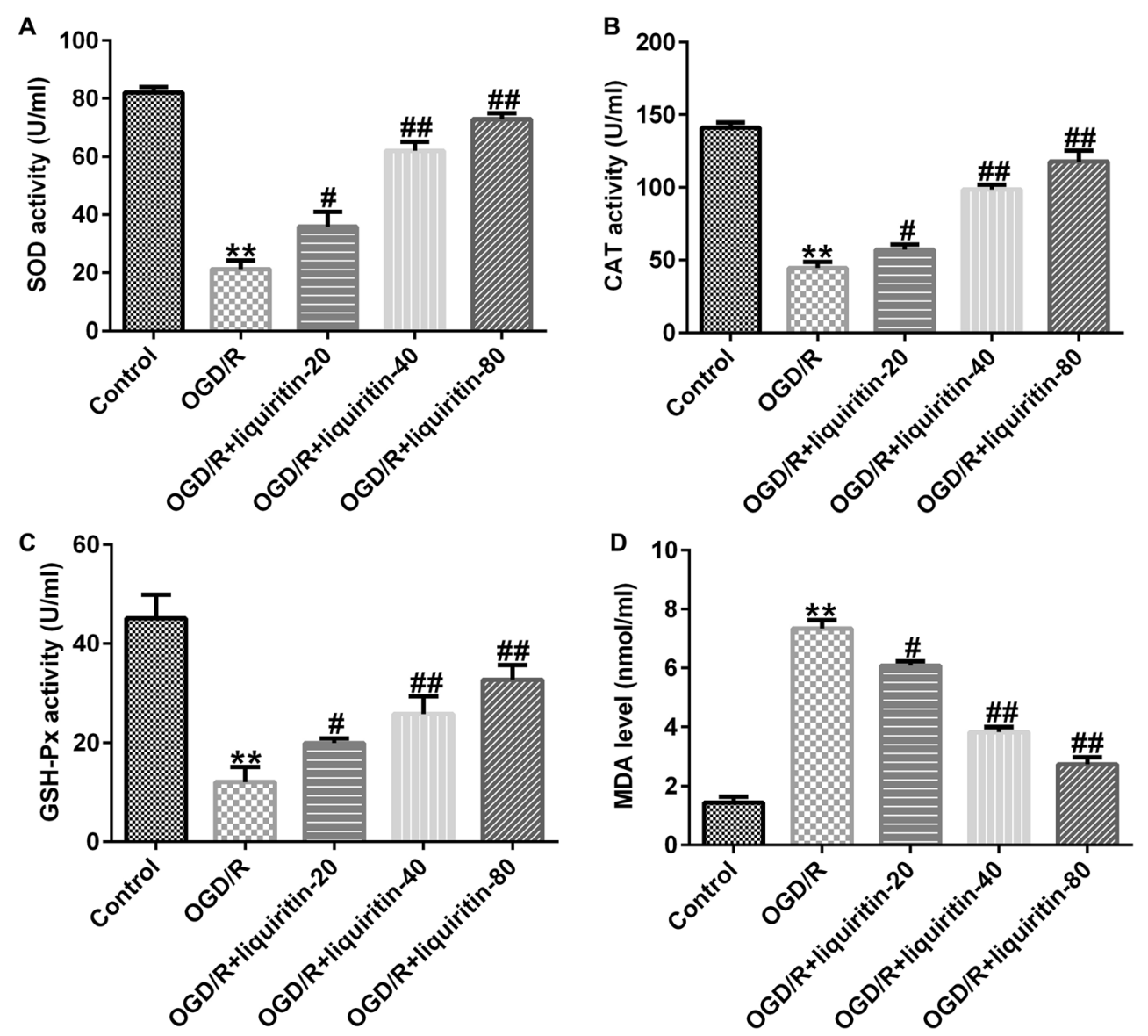

min. Subsequently, total protein was quantified using a BCA protein assay kit (Beyotime Institute of Biotechnology). Proteins were boiled at $95{ }^{\circ} \mathrm{C}$ for $10 \mathrm{~min}$, and a total of $20 \mu \mathrm{g}$ protein was separated by sodium dodecyl sulfatepolyacrylamide gel electrophoresis (SDS-PAGE) on a $10 \%$ gel. The separated proteins were subsequently transferred to polyvinylidene fluoride (PVDF) membranes and blocked for $1 \mathrm{~h}$ at room temperature with $5 \%$ non-fat milk. The membranes were incubated with primary antibodies against Bcl-2 (CST Biological Reagents Co., Ltd.; 1:1,000), Bax (CST Biological Reagents Co., Ltd.; 1:1,000), phosphorylated (p)-p65 (CST Biological Reagents Co., Ltd.; 1:1,000), p65 (CST Biological Reagents Co., Ltd.; 1:1,000), p-p38 (CST Biological Reagents Co., Ltd.; 1:1,000), p38 (CST Biological Reagents Co., Ltd.; 1:1,000), and GAPDH (Santa Cruz Biotechnology, Inc.; $1: 1,000)$ at $4{ }^{\circ} \mathrm{C}$ overnight. Following primary incubation,

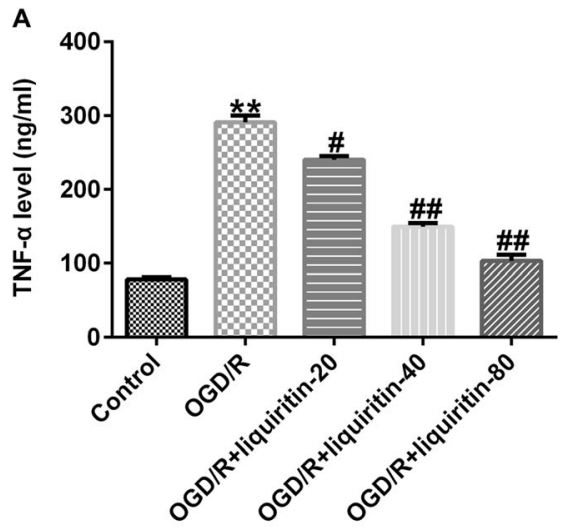

Fig. 4 Liquiritin decreases the expression levels of inflammatory factors in SH-SY5Y cells induced by OGD/R. ELISA was used to detect the expression levels of (A) TNF- $\alpha$, (B) IL-1 $\beta$, and (C) IL-6 in OGD/R-
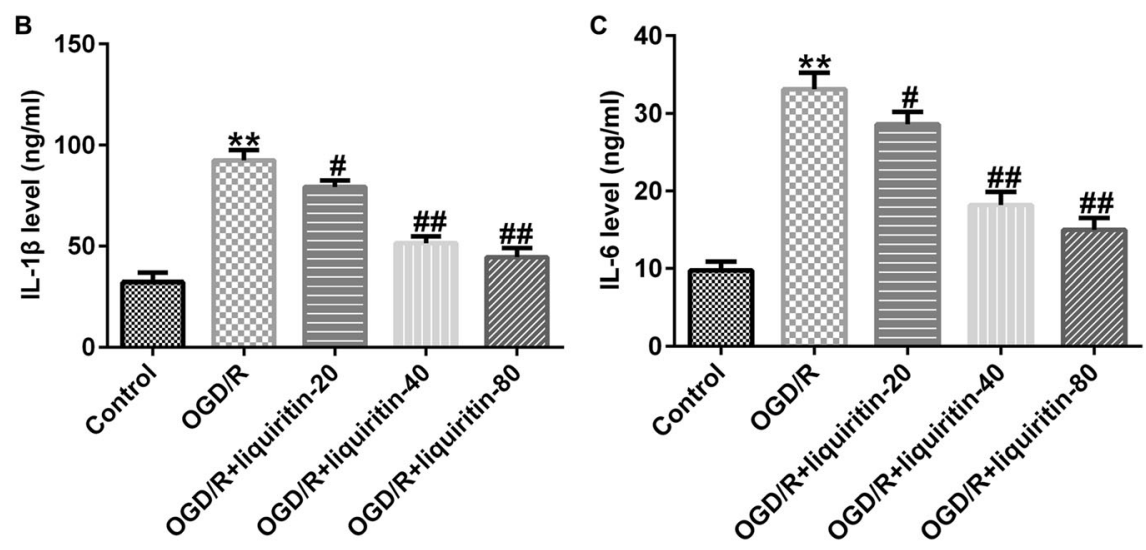

induced SH-SY5Y cells in different groups. Data are presented as the mean \pm SD. ${ }^{* *} p<0.01$ vs. control; ${ }^{\#} p<0.05,{ }^{\# \#} p<0.01$ vs. OGD/R. Abbreviation: OGD/R, oxygen and glucose deprivation/reoxygenation 
membranes were incubated with HRP-conjugated secondary antibodies $(1: 1,000)$ for $1 \mathrm{~h}$. Protein bands were visualized using the enhanced chemiluminescence (ECL) detection system.

\section{RT-qPCR Analysis}

To isolate RNA from SH-SY5Y cells, a TRIzol reagent (Thermo Fisher Scientific, Inc.) was used according to the manufacturer's instructions. To transcribe total RNA into cDNA, a PrimeScript RT kit (Takara Bio, Inc.) was used. The temperature protocol for the reverse transcription reaction was as follows: $25^{\circ} \mathrm{C}$ for $5 \mathrm{~min}, 42{ }^{\circ} \mathrm{C}$ for 60 $\mathrm{min}$, and $80^{\circ} \mathrm{C}$ for $2 \mathrm{~min}$. To examine the mRNA expression levels of Bcl-2 and Bax, qPCR amplification was then applied with SYBR Premix Ex-Taq (Takara Bio, Inc.) according to the manufacturer's instructions. The thermocycling conditions were as follows: Initial denaturation at 95 ${ }^{\circ} \mathrm{C}$ for $5 \mathrm{~min}$, followed by 40 cycles of denaturation at 95 ${ }^{\circ} \mathrm{C}$ for $15 \mathrm{~s}$ and annealing/elongation at $60{ }^{\circ} \mathrm{C}$ for $30 \mathrm{~s}$. $\beta$ Actin was used as the internal control. Primers were listed as follows: $\beta$-actin-forward, 5'-GAGCACAGAGCCTC GCCTTT-3' and reverse, 5'-GCCCACATAGGAAT CCTTCTG-3'; Bcl-2 forward, 5'-AGGATTGTGGCCTT CTTTGAG-3' and reverse, 5'-AGCCAGGAGAAATC AAACAGAG-3' and Bax forward, 5'-TCTG AGCAGATCATGAAGACAGG-3' and reverse, 5'-ATCC TCTGCAGCTCCATGTTAC- ${ }^{\prime}$. To calculate the gene expression, the $2^{-\Delta \Delta C q}$ method (Livak and Schmittgen 2001) was performed.

\section{Statistical Analysis}

All data are presented as the mean \pm standard deviation. Data were analyzed using SPSS 17.0 software (SPSS, Inc.). Statistical differences between two groups were measured with Student's $t$-tests, and differences between multiple groups were measured by application of one-way ANOVA; $p<0.05$ was considered to indicate a statistically significant difference.

\section{Results and Discussion}

\section{Effects on Cell Viability}

As shown in Fig. 1A and B, the results of the MTT and LDH assays demonstrated that there was no significant difference between the liquiritin (1) treatment and control groups at different time points. Thus, 0-, 20-, 40-, and $80-\mu \mathrm{M}$ liquiritin concentrations were used for subsequent experiments.

\section{Survival and Apoptosis of OGD/R-Induced SH-SY5Y Cells}

We then explored the functions of liquiritin (1) in an OGD/R model of SH-SY5Y cells. Results of the present study demonstrated that cell viability was reduced, and the levels of LDH activity and cell apoptosis were upregulated in the OGD/R treatment group, compared with the control group (Fig. 2A-D). In addition, the expression levels of Bax were upregulated, and the expression levels of Bcl-2 were downregulated in OGD/R-treated cells compared with the control group (Fig. 2E, F). The aforementioned findings were reversed following treatment with liquiritin in a dose-dependent manner. Collectively, these results demonstrated that liquiritin promoted the viability and reduced the apoptosis of OGD/R-treated SH-SY5Y cells.

\section{Oxidative Stress}

Oxidative stress is a key pathological marker for several neurological diseases, including Alzheimer's disease
A

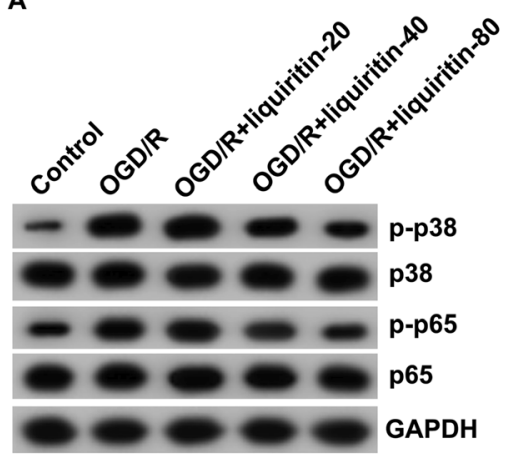

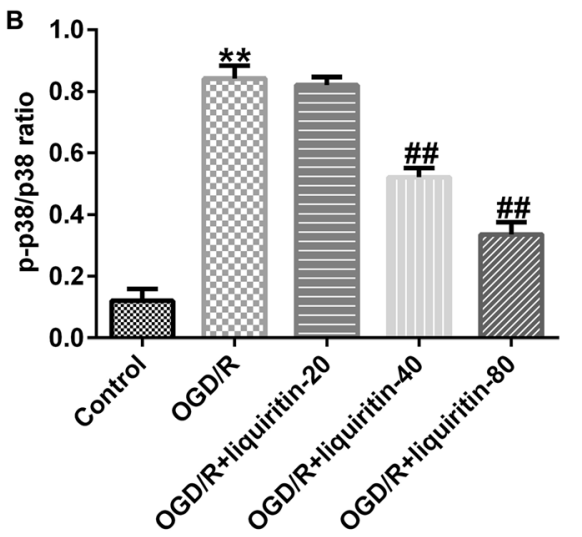

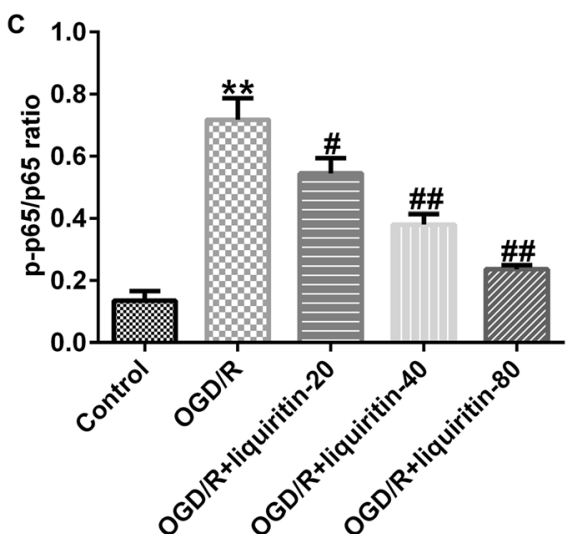

Fig. 5 Liquiritin inhibits activation of the p38MAPK/NF- $k B$ signaling pathway in SH-SY5Y cells induced by OGD/R. (A-C) Expression levels of p-p38 and p-p65, and the ratios of p-p38/p38 and p-p65/p65 were determined using western blot analyses. Data are presented as the mean $\pm \mathrm{SD} .{ }^{* *} p<0.01$ vs. control; ${ }^{*} p<0.05,{ }^{\# \#} p<0.01 v s$. OGD $/ \mathrm{R}$. Abbreviations: OGD/R, oxygen and glucose deprivation/reoxygenation; p, phosphorylated 
(Chen et al. 2012). It has previously been established that a redox imbalance is also a key characteristic associated with $\mathrm{OGD} / \mathrm{R}$ injury. The results of the present study demonstrated that the levels of SOD, CAT, and GSH-Px were decreased, and the levels of MDA were increased in OGD/ R-treated cells, compared with the control. However, treatment with liquiritin (1) increased the levels of SOD, CAT, and GSH-Px, and reduced the levels of MDA in a dosedependent manner compared with the OGD/R group (Fig. $3 \mathrm{~A}-\mathrm{D})$. Collectively, these results revealed that treatment with liquiritin promoted the antioxidant activity of OGD/
R-treated SH-SY5Y cells, and further reduced oxidative damage. Collectively, the findings of the present study, and those of previous studies (Cai et al. 2020), revealed that liquiritin may restore oxidant/antioxidant balance and inhibit injury caused by $\mathrm{I} / \mathrm{R}$ damage.

\section{Inhibition of Inflammatory Response}

Previously, it has been reported that activation of NF-KB is associated with increased levels of IL- $1 \beta$ and TNF- $\alpha$ following cerebral I/R injury, and suppression of NF- $\mathrm{kB}$ may contribute to
A

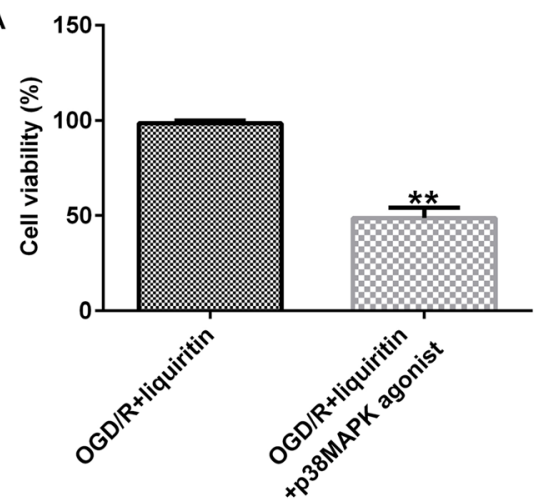

C

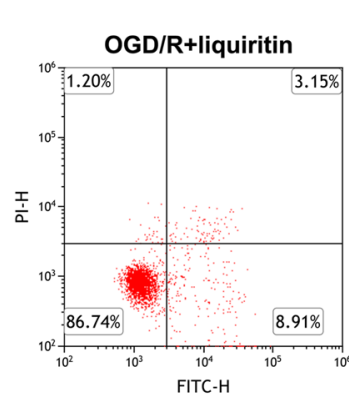

B

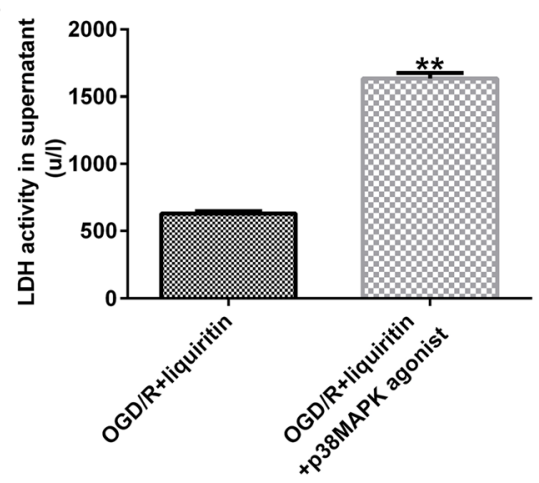

D

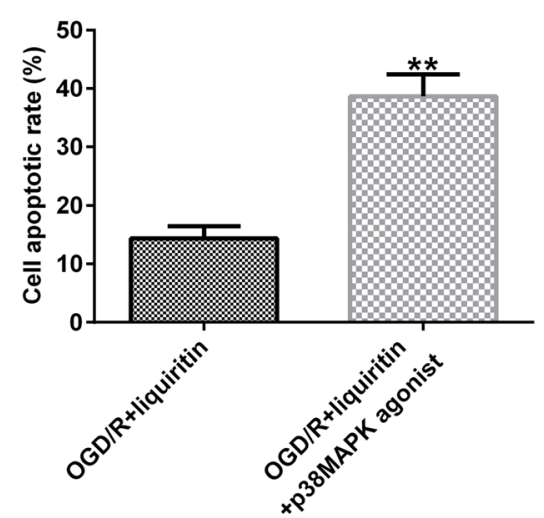

E

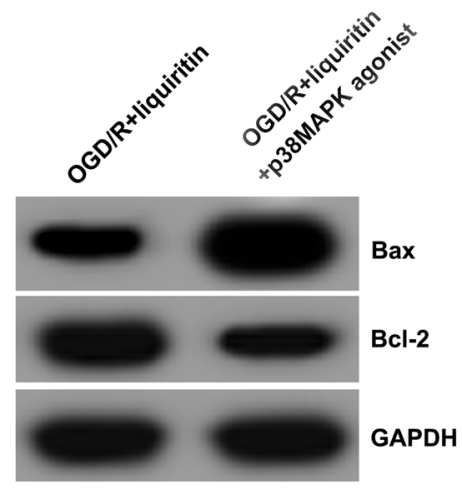

OGD/R+liquiritin

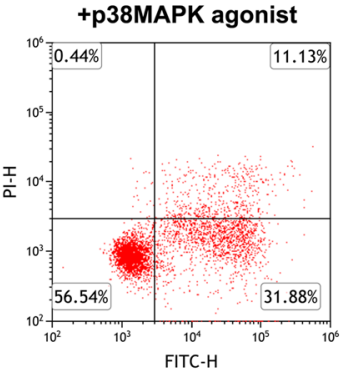

$\mathrm{F}$

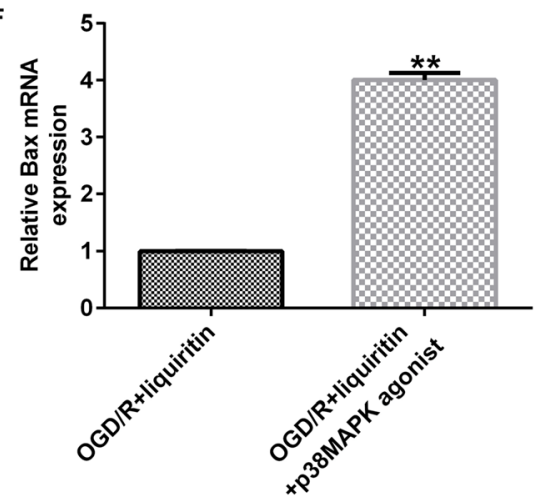

G

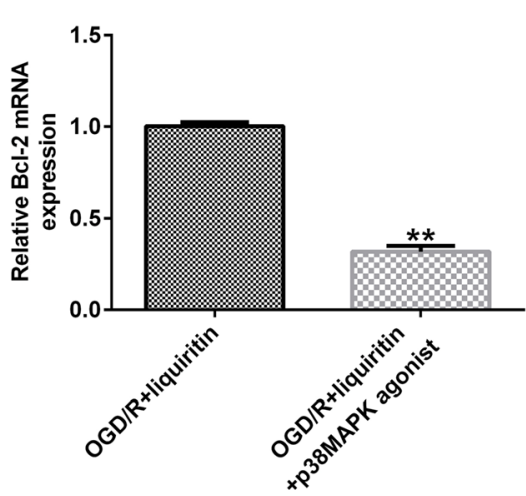

Fig. 6 Treatment with a p38/MAPK agonist reverses the liquiritininduced effects on the viability and apoptosis of SH-SY5Y cells induced by OGD/R. SH-SY5Y cells were divided into two groups: (i) OGD/R + liquiritin $(80 \mu \mathrm{M})$ and (ii) $\mathrm{OGD} / \mathrm{R}+$ liquiritin $(80 \mu \mathrm{M})+\mathrm{p} 38 / \mathrm{MAPK}$ agonist (P79350; $50 \mu \mathrm{M})$ groups. (A and B) Cell viability and LDH activity were investigated using MTT and LDH assays, respectively. (C and D) Cell apoptosis was determined using flow cytometry assays. (E) Western blot analysis was used to detect the expression levels of Bax and Bcl-2. (F and $\mathbf{G}$ ) mRNA expression levels of Bax and Bcl-2. Data are presented as the mean $\pm \mathrm{SD}$. $* * p<0.01 v s$. OGD $/ \mathrm{R}+$ liquiritin. Abbreviations: OGD/R, oxygen and glucose deprivation/reoxygenation; $\mathrm{LDH}$, lactate dehydrogenase 

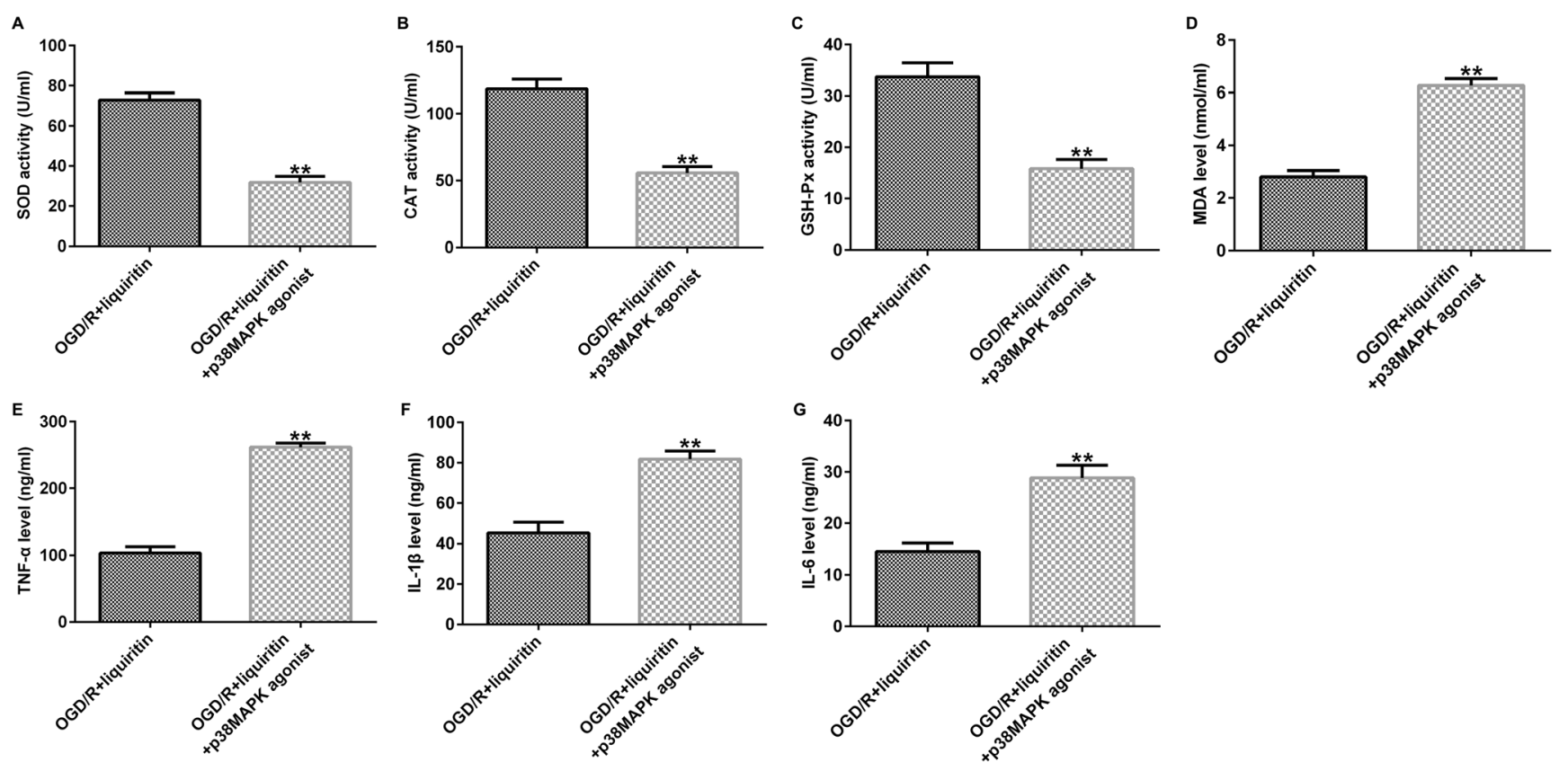

Fig. 7 Treatment with a p38/MAPK agonist reverses the inhibitory effects of liquiritin on OGD/R-induced oxidative stress and inflammatory responses in SH-SY5Y cells. (A-D) Indicators of antioxidant enzyme activity, such as SOD, CAT, and GSH-Px, and MDA expression levels were detected using commercially available kits. ELISA was used to detect the expression levels of (E) TNF- $\alpha$, (F) IL-1 $\beta$, and (G) IL-6 in

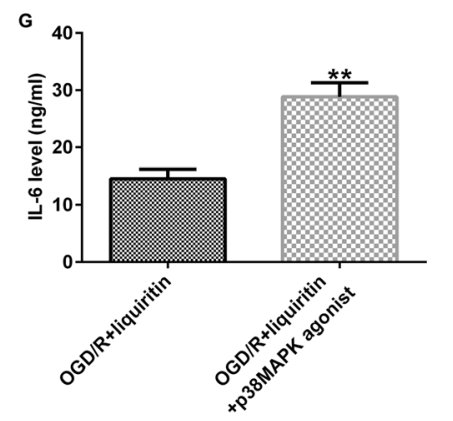

OGD/R-induced SH-SY5Y cells in different groups. Data are presented as the mean $\pm \mathrm{SD} .{ }^{* *} p<0.01$ vs. $\mathrm{OGD} / \mathrm{R}+$ liquiritin. Abbreviations: OGD/R, oxygen and glucose deprivation/reoxygenation; SOD, superoxide dismutase; CAT, catalase; GSH-Px, glutathione peroxidase; MDA, malondialdehyde

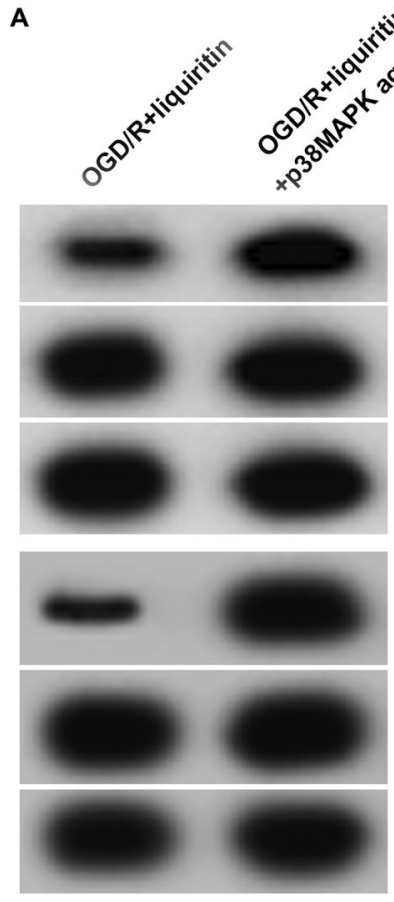

Fig. 8 Treatment with a p38/MAPK agonist reverses the inhibitory effects of liquiritin on the p38MAPK/NF-KB signaling pathway in OGD/Rinduced SH-SY5Y cells. Protein expression levels of (A) p-p38 and p$\mathrm{p} 65$, and the (B) p-p38/p38 and (C) p-p65/p65 ratios were determined

GAPDH
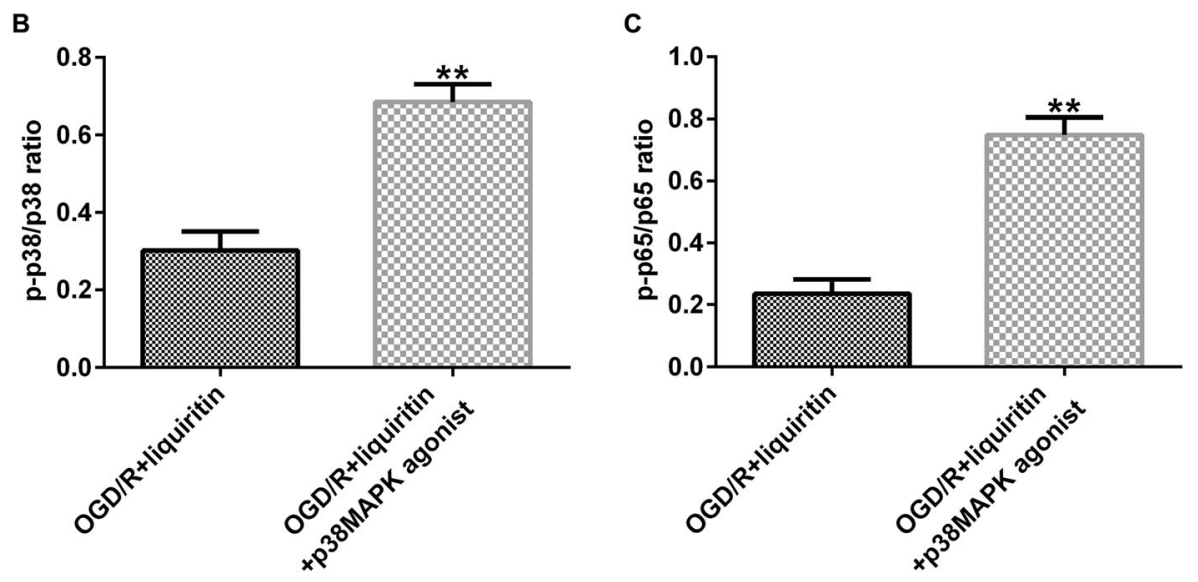

using western blot analyses. Data are presented as the mean $\pm \mathrm{SD} .{ }^{* *} p<$ 0.01 vs. OGD/R + liquiritin. Abbreviations: $\mathrm{OGD} / \mathrm{R}$, oxygen and glucose deprivation/reoxygenation; $\mathrm{p}$, phosphorylated 
the protection of the heart from ischemic damage (Li et al. 2014). In the present study, ELISA results demonstrated that levels of TNF- $\alpha$, IL- $1 \beta$, and IL- 6 were significantly increased in the cell supernatant for the OGD/R group as compared with the control group. In addition, levels of TNF- $\alpha$, IL- $1 \beta$, and IL- 6 were decreased following treatment with liquiritin (1) in a dosedependent manner (Fig. 4A-C).

\section{p38MAPK/NF-kB Signaling Pathway}

To verify the molecular mechanisms for liquiritin (1) action in OGD/R-induced injury, markers associated with the p38MAPK/ NF-KB signaling pathway were detected. Following cerebral I/R injury, activation of the p38/MAPK signaling pathway is increased, and inhibition of p38/MAPK may protect neurons from injury (Zhen et al. 2016). Results from a previous study reported that liquiritin protected high-fructose-induced myocardial fibrosis by suppressing the NF-KB/MAPK signaling pathway (Zhang et al. 2016). Treatment with liquiritin also reduced rheumatoid arthritis by reducing inflammation, inhibiting angiogenesis, and suppressing the MAPK signaling pathway (Zhai et al. 2019). In the present investigation, the results of the western blot analysis demonstrated that the expression levels of p-p65 and p-p38, and the ratios of p-p65/p65 and p-p38/p38 were markedly increased in the OGD/R group compared with the control group. However, the expression levels of p-p38 and p-p65, and the p-p38/p38 and p-p65/p65 ratios were markedly decreased following treatment with liquiritin in a dose-dependent manner, compared with the OGD/R group (Fig. 5A-C). Collectively, these findings demonstrated that the MAPK/NF- $\mathrm{kB}$ signaling pathway played a role in OGD/R-treated SH-SY5Y cells.

The association between liquiritin and the MAPK/NF-KB signaling pathway in OGD/R-induced SH-SY5Y cell injury was analyzed. The results of the present study demonstrated that cell viability was reduced, LDH activity was increased, and cell apoptosis was enhanced in the OGD/R + liquiritin + p38/MAPK agonist group, compared with the OGD/R + liquiritin group (Fig. 6A-D). Moreover, the levels of Bcl-2 were reduced, and the levels of Bax were increased in the $\mathrm{OGD} / \mathrm{R}+$ liquiritin + p38/MAPK agonist group, compared with those in the OGD/R + liquiritin group (Fig. 6E-G).

To further determine the association between liquiritin and the MAPK/NF- $\mathrm{KB}$ signaling pathway in OGD/R-induced oxidative stress, key indicators of oxidative stress were detected. The levels of SOD, CAT, and GSH-Px were increased, and the levels of MDA were decreased in the OGD/R + liquiritin + p38/MAPK agonist group, compared with those in the $\mathrm{OGD} / \mathrm{R}+$ liquiritin group (Fig. 7A-D). Moreover, the levels of TNF- $\alpha$, IL-1 $\beta$, and IL-6 were significantly increased in the cell supernatant of the $\mathrm{OGD} / \mathrm{R}$ group, compared with the $\mathrm{OGD} / \mathrm{R}+$ liquiritin treatment group (Fig. 7E-G). Collectively, these results demonstrated that treatment with the p38/MAPK agonist reversed the inhibitory effects of liquiritin on OGD/R-induced oxidative stress and the inflammatory response in SH-SY5Y cells.

Subsequently, the association between liquiritin and the p38/MAPK agonist was investigated in OGD/R-induced SH-SY5Y cells. Compared with the OGD/R + liquiritin group, the expression levels of $\mathrm{p}-\mathrm{p} 38$ and $\mathrm{p}-\mathrm{p} 65$, and the $\mathrm{p}$ $\mathrm{p} 38 / \mathrm{p} 38$ and $\mathrm{p}-\mathrm{p} 65 / \mathrm{p} 65$ ratios were significantly increased in the OGD/R + liquiritin + p38/MAPK agonist group (Fig. 8AC). Collectively, these results demonstrated that treatment with liquiritin reduced neuronal injury induced by $\mathrm{OGD} / \mathrm{R}$ via repression of the MAPK/NF- $\mathrm{KB}$ signaling pathway.

\section{Conclusion}

In conclusion, this study demonstrated that treatment with liquiritin relieved OGD/R-induced neuronal damage via suppression of the $\mathrm{p} 38 \mathrm{MAPK} / \mathrm{NF}-\mathrm{kB}$ signaling pathway. Thus, the findings of the present study highlighted that liquiritin may serve as a novel agent for the treatment of cerebral I/R injury. This research provides additional basis for a novel strategy in the clinical treatment of cerebral I/R injury.

Author Contribution LL contributed to the study design, data collection, statistical analysis, data interpretation, and manuscript preparation. ZZ contributed to data collection, statistical analysis, and manuscript preparation. All authors read and approved the final manuscript.

Funding The present study was supported by the Key Projects of Hunan Province Chinese Medicine Research Project (grant no. 202015), the Key Projects of Scientific Research Project of Hunan Health Committee (grant no. 20200308), and the General Program of Hunan Provincial Natural Science Foundation of China (NO.2021JJ30529).

\section{Declarations}

Competing Interests The authors declare no competing interests.

Open Access This article is licensed under a Creative Commons Attribution 4.0 International License, which permits use, sharing, adaptation, distribution and reproduction in any medium or format, as long as you give appropriate credit to the original author(s) and the source, provide a link to the Creative Commons licence, and indicate if changes were made. The images or other third party material in this article are included in the article's Creative Commons licence, unless indicated otherwise in a credit line to the material. If material is not included in the article's Creative Commons licence and your intended use is not permitted by statutory regulation or exceeds the permitted use, you will need to obtain permission directly from the copyright holder. To view a copy of this licence, visit http://creativecommons.org/licenses/by/4.0/.

\section{References}

Abdel-Rahman RF, Alqasoumi SI, Ogaly HA, Abd-Elsalam RM, ElBanna HA, Soliman GA (2020) Propolis ameliorates cerebral injury 
in focal cerebral ischemia/reperfusion (I/R) rat model via upregulation of TGF-beta 1. Saudi Pharm J 28:116-126. https://doi.org/10. 1016/j.jsps.2019.11.013

Cai HA, Tao X, Zheng LJ, Huang L, Peng Y, Liao RY, Zhu YM (2020) Ozone alleviates ischemia/reperfusion injury by inhibiting mitochondrion-mediated apoptosis pathway in SH-SY5Y cells. Cell Biol Int 44:975-984. https://doi.org/10.1002/cbin.11294

Chen SD, Yin JH, Hwang CS, Tang CM, Yang DI (2012) Anti-apoptotic and anti-oxidative mechanisms of minocycline against sphingomyelinase/ceramide neurotoxicity: implication in Alzheimer's disease and cerebral ischemia. Free Radical Res 46: 940-950. https://doi.org/10.3109/10715762.2012.674640

Geng Y, Chen Y, Sun W, Gu Y, Zhang Y, Li M, Xie J, Tian X (2020) Electroacupuncture ameliorates cerebral I/R-induced inflammation through DOR-BDNF/TrkB pathway. Evid-Based Compl Alt 2020: 3495836. https://doi.org/10.1155/2020/3495836

He J, Zhang Y, Xu T, Zhao Q, Wang D, Chen CS, Tong W, Liu C, Xu T, Ju Z, Peng Y (2014) Effects of immediate blood pressure reduction on death and major disability in patients with acute ischemic stroke: the CATIS randomized clinical trial. J Am Med Assoc 311:479 489. https://doi.org/10.1001/jama.2013.282543

Jia SL, Wu XL, Li XX, Dai XL, Gao ZL, Lu Z, Zheng QS, Sun YX (2016) Neuroprotective effects of liquiritin on cognitive deficits induced by soluble amyloid-beta(1-42) oligomers injected into the hippocampus. J Asian Nat Prod Res 18:1186-1199. https://doi. org/10.1080/10286020.2016.1201811

Li YW, Zhang Y, Zhang L, Li X, Yu JB, Zhang HT, Tan BB, Jiang LH, Wang YX, Liang Y, Zhang XS, Wang WS, Liu HG (2014) Protective effect of tea polyphenols on renal ischemia/reperfusion injury via suppressing the activation of TLR4/NF-kappa B p65 signal pathway. Gene 542:46-51. https://doi.org/10.1016/j.gene.2014. 03.021

Li XQ, Cai LM, Liu J, Ma YL, Kong YH, Li H, Jiang M (2018) Liquiritin suppresses UVB-induced skin injury through prevention of inflammation, oxidative stress and apoptosis through the TLR4/MyD88/ NF-kappa B and MAPK/caspase signaling pathways. Int J Mol Med 42:1445-1459. https://doi.org/10.3892/ijmm.2018.3720

Li YF, Fei LP, Wang JL, Niu QY (2020a) Inhibition of miR-217 protects against myocardial ischemia-reperfusion injury through inactivating NF-kappa B and MAPK pathways. Cardiovasc Eng Techn 11:219227. https://doi.org/10.1007/s13239-019-00452-z

Li X, Qin X, Tian J, Gao X, Wu X, Du G, Zhou Y (2020b) Liquiritin protects $\mathrm{PC} 12$ cells from corticosterone-induced neurotoxicity via regulation of metabolic disorders, attenuation ERK1/2-NF-kappa B pathway, activation Nrf2-Keap1 pathway, and inhibition mitochondrial apoptosis pathway. Food Chem Toxicol 146:111801. https:// doi.org/10.1016/j.fct.2020.111801

Liao SC, Apaijai N, Chattipakorn N, Chattipakorn SC (2020) The possible roles of necroptosis during cerebral ischemia and ischemia/ reperfusion injury. Arch Biochem Biophys 695:108629. https:// doi.org/10.1016/j.abb.2020.108629
Liu S, Dai Q, Hua R, Liu T, Han S, Li S, Li J (2017) Determination of brain-regional blood perfusion and endogenous cPKC gamma impact on ischemic vulnerability of mice with global ischemia. Neurochem Res 42:2814-2825. https://doi.org/10.1007/s11064017-2294-9

Liu H, Wu X, Luo J, Zhao L, Li X, Guo H, Bai H, Cui W, Guo W, Feng D, Qu Y (2020) Adiponectin peptide alleviates oxidative stress and NLRP3 inflammasome activation after cerebral ischemiareperfusion injury by regulating AMPK/GSK-3 beta. Exp Neurol 329:113302. https://doi.org/10.1016/j.expneurol.2020.113302

Livak KJ, Schmittgen TD (2001) Analysis of relative gene expression data using real-time quantitative PCR and the $2-\Delta \Delta \mathrm{Cq}$ method. Methods 25:402-408. https://doi.org/10.1006/meth.2001.1262

Meng CC, Zhang JY, Zhang LY, Wang YT, Li ZY, Zhao J (2019) Effects of NLRP6 in cerebral ischemia/reperfusion (I/R) injury in rats. J Mol Neurosci 69:411-418. https://doi.org/10.1007/s12031-019-01370-4

Xie W, Zhu T, Dong X, Nan F, Meng X, Zhou P, Sun G, Sun X (2019) HMGB1-triggered inflammation inhibition of notoginseng leaf triterpenes against cerebral ischemia and reperfusion injury via MAPK and NF-kappa B signaling pathways. Biomolecules 9:512. https://doi.org/10.3390/biom9100512

Xu M, Wu R, Liang Y, Fu K, Zhou Y, Li X, Wu L, Wang Z (2020) Protective effect and mechanism of Qishiwei Zhenzhu pills on cerebral ischemia-reperfusion injury via blood-brain barrier and metabonomics. Biomed Pharmacother 131:110723. https://doi.org/10. 1016/j.biopha.2020.110723

Zhai KF, Duan H, Cui CY, Cao YY, Si JL, Yang HJ, Wang YC, Cao WG, Gao GZ, Wei ZJ (2019) Liquiritin from Glycyrrhiza uralensis attenuating rheumatoid arthritis via reducing inflammation, suppressing angiogenesis, and inhibiting MAPK signaling pathway. $\mathrm{J}$ Agr Food Chem 67:2856-2864. https://doi.org/10.1021/acs.jafc. 9b00185

Zhang YY, Zhang YP (2020) lncRNA ZFAS1 improves neuronal injury and inhibits inflammation, oxidative stress, and apoptosis by sponging miR-582 and upregulating NOS3 expression in cerebral ischemia/reperfusion injury. inflammation 43:1337-1350. https:// doi.org/10.1007/s10753-020-01212-1

Zhang Y, Zhang L, Zhang Y, Xu JJ, Sun LL, Li SZ (2016) The protective role of liquiritin in high fructose-induced myocardial fibrosis via inhibiting NF-kappa B and MAPK signaling pathway. Biomed Pharmacother 84:1337-1349. https://doi.org/10.1016/j.biopha. 2016.10.036

Zhen YL, Ding CJ, Sun JQ, Wang YN, Li S, Dong LY (2016) Activation of the calcium-sensing receptor promotes apoptosis by modulating the JNK/p38 MAPK pathway in focal cerebral ischemia-reperfusion in mice. Am J Transl Res 8:911-921

Zhou Y, Jiao YH, Sun YH, Gao SM (2020) In vitro production and distribution of flavonoids in Glycyrrhiza uralensis Fisch. J Food Sci Tech Mys 57:1553-1564. https://doi.org/10.1007/s13197-01904191-w 